\title{
The whole picture
}

\section{As the techniques for imaging whole animals become more sophisticated, researchers are able to get a clearer picture of what is going on inside. Monya Baker looks at the options available.}

Dead mice tell too few tales. Conventional animal imaging requires sacrificing multiple animals at numerous time points, then slicing and staining tissue to identify the location and state of particular molecules at a point in time. However, it would be easier and more instructive if researchers could image the whole body to follow the biological processes within a live animal, ideally with no need for surgery or other invasive techniques. There are many established technologies for human imaging that work in animals, but advances in optical imaging in particular are providing fresh opportunities to see inside smaller creatures.

Such advances are allowing researchers to follow disease progression and drug response more precisely than they can from dissecting organs, says David Piwnica-Worms, who directs the molecular imaging centre at Washington University in Saint Louis, Missouri. "Because you can use each animal as its own control, something that would have been lost in the noise becomes very, very clear." And whole-body imaging means not just better data, but also new types of data. For example, disease processes often start well before symptoms become evident. Last year, using new optical imaging technologies, researchers led by Stanley Prusiner at the University

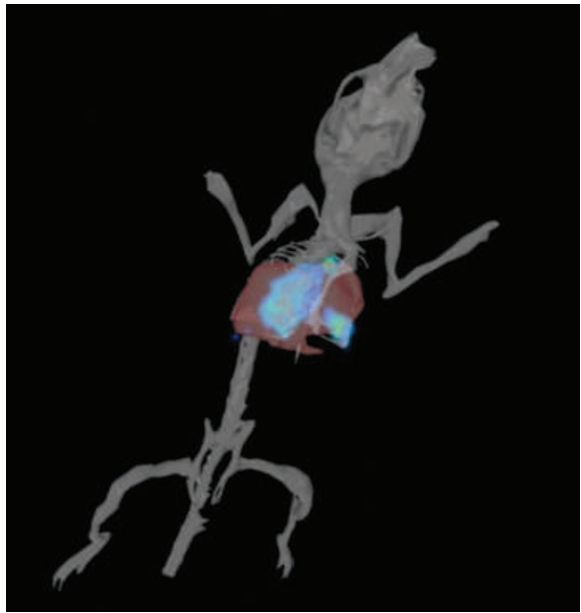

By combining modalities, researchers can pinpoint where processes are taking place.

of California, San Francisco, detected the onset of a mouse equivalent of neurodegenerative conditions such as Creutzfeldt-Jakob disease nearly two months before behaviour was affected ${ }^{1}$. Rather than comparing brains of animals killed at various stages of disease, the researchers could watch the prion disease spread through the brains of individual animals. Researchers can also monitor other processes: T cells travelling to inflamed sites, 움 tumours spreading or shrinking, even enzymes catalysing reactions within cells.

A diverse range of imaging techniques, or modalities, is now available (see Table). Magnetic resonance imaging (MRI), positron electron tomography (PET), computed tomography (CT) and single-photon emission computed tomography (SPECT) are all used routinely to scan patients in hospitals; smaller, less expensive versions have been produced for animal research. These techniques can penetrate deep into tissue, and sources of distortion are relatively few and largely understood. They are often used to survey whole bodies for disease and to do cross-sectional imaging, particularly for research on deep-seated organs.

Optical techniques are used to probe morelocal processes and can readily make use of multiple labels, or signal-emitting tags that are attached to a molecule of interest, such as a fluorescent dye on an antibody. The downside is that light is scattered and absorbed quickly within the body, complicating attempts to quantify signals and limiting imaging ability to a couple of centimetres or so below the skin surface. "No single modality has the lock on being the best molecular strategy for wholeanimal imaging under all circumstances.

\section{PROS AND CONS OF IMAGING MODALITIES}

\begin{tabular}{|c|c|c|c|c|c|c|c|c|}
\hline Technique & Labels & Signal measured & Strengths & Weaknesses & Cost & Throughput & $\begin{array}{l}\text { Sensitivity (moles } \\
\text { of label detected) }\end{array}$ & Resolution \\
\hline PET & $\begin{array}{l}\text { Radiolabelled } \\
\text { molecules }\end{array}$ & $\begin{array}{l}\text { Positrons from } \\
\text { radionuclides }\end{array}$ & Highly sensitive & $\begin{array}{l}\text { Can detect only one } \\
\text { radionuclide, requires } \\
\text { radioactivity }\end{array}$ & High & Low & $10^{-15}$ & $1-2 \mathrm{~mm}$ \\
\hline SPECT & $\begin{array}{l}\text { Radiolabelled } \\
\text { molecules }\end{array}$ & $\gamma$-rays & $\begin{array}{l}\text { Can distinguish } \\
\text { between radionuclides, } \\
\text { so more processes can } \\
\text { be imaged at once }\end{array}$ & Requires radioactivity & High & Low & $10^{-14}$ & $1-2 \mathrm{~mm}$ \\
\hline $\mathrm{CT}$ & None & X-rays & $\begin{array}{l}\text { Fast, cross-sectional } \\
\text { images }\end{array}$ & $\begin{array}{l}\text { Poor resolution of } \\
\text { soft tissues }\end{array}$ & High & Low & $10^{-6}$ & $50 \mu \mathrm{m}$ \\
\hline MRI & $\begin{array}{l}\text { Can use isotope- } \\
\text { labelled molecular } \\
\text { tracers }\end{array}$ & $\begin{array}{l}\text { Alterations in } \\
\text { magnetic fields }\end{array}$ & $\begin{array}{l}\text { Harmless, high- } \\
\text { resolution of soft tissues }\end{array}$ & $\begin{array}{l}\text { Cannot follow many } \\
\text { labels }\end{array}$ & High & Low & $10^{-9}-10^{-6}$ & $50 \mu \mathrm{m}$ \\
\hline Optical & $\begin{array}{l}\text { Genetically engineered } \\
\text { proteins and } \\
\text { bioluminescent and } \\
\text { fluorescently labelled } \\
\text { probes }\end{array}$ & $\begin{array}{l}\text { Light, } \\
\text { particularly in } \\
\text { the infrared }\end{array}$ & $\begin{array}{l}\text { Easy, non-damaging } \\
\text { technique readily } \\
\text { adapted to study } \\
\text { specific molecular } \\
\text { events }\end{array}$ & $\begin{array}{l}\text { Poor depth } \\
\text { penetration }\end{array}$ & Low & High & $10^{-12}$ & $1-2 \mathrm{~mm}$ \\
\hline Photoacoustic & $\begin{array}{l}\text { Probes that absorb } \\
\text { light and create sound } \\
\text { signals }\end{array}$ & Sound & $\begin{array}{l}\text { Better depth resolution } \\
\text { than light }\end{array}$ & $\begin{array}{l}\text { Information } \\
\text { processing and } \\
\text { machines still being } \\
\text { optimized }\end{array}$ & Low & High & $10^{-12}$ & $50 \mu \mathrm{m}$ \\
\hline Ultrasound & $\begin{array}{l}\text { Microbubbles, which } \\
\text { can be combined with } \\
\text { targeted contrast } \\
\text { agents }\end{array}$ & Sound & Quick, harmless & $\begin{array}{l}\text { Poor image contrast, } \\
\text { works poorly in air- } \\
\text { containing organs }\end{array}$ & Low & High & $10^{-8}$ & $50 \mu \mathrm{m}$ \\
\hline
\end{tabular}




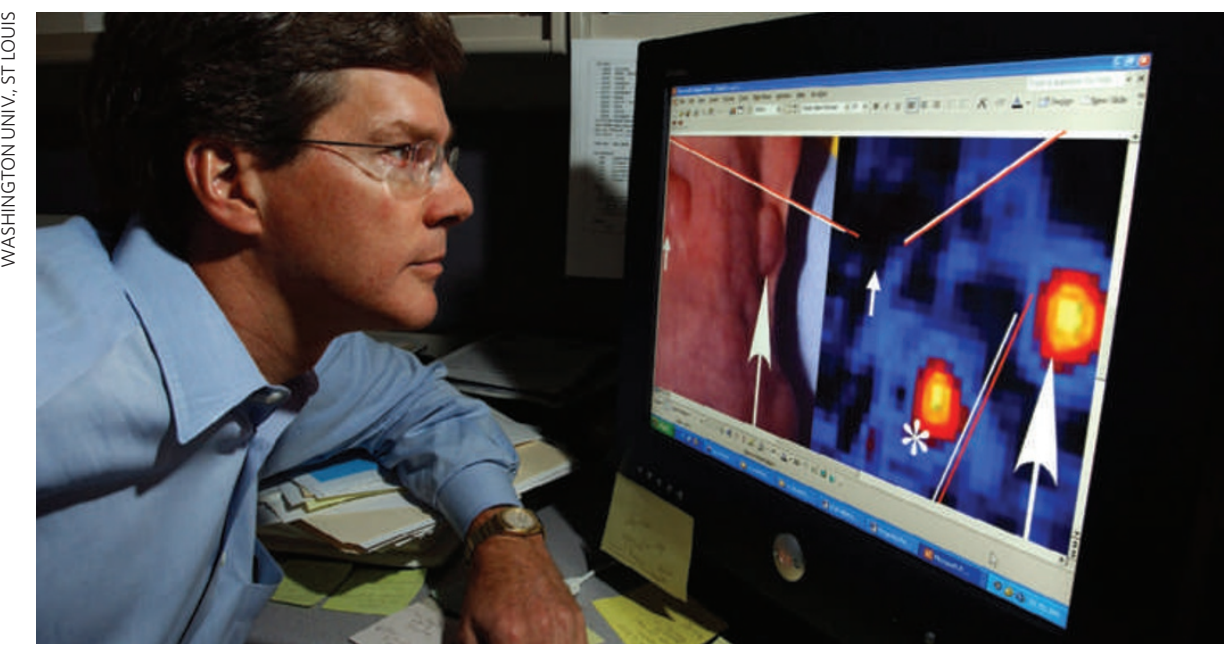

David Piwnica-Worms has developed new bioluminescent techniques as well as ways to combine bioluminescence with magnetic resonance imaging.

Different biological queries require different strategies," says Piwnica-Worms. "The different modalities have strengths that apply in one niche but not in another."

As a field, whole-animal imaging has come a long way since the 1990s, when vendors began offering mouse-sized versions of their instruments to lure customers from research hospitals, and when those selling systems to read labelled gels started to adapt their systems to image mice.

\section{Fast, cheap and multicoloured}

Optical techniques have probably been the fastest growing way to image small animals. At the US National Cancer Institute's Small Animal Imaging Resource Program, twice as many funding recipients bought optical equipment as bought MRI, PET or SPECT machines for imaging small animals from 1999 to 2007. Programme director Barbara Croft says that much of this can be explained by the fact that optical instruments are perhaps a quarter of the cost of the other imaging technologies, and are also easy to use. "People who don't know anything else about small-animal imaging are more likely to buy optical equipment than anything else," she says.

There are two main approaches to optical imaging. In fluorescent techniques, labels introduced into an animal give off light of one wavelength when excited by light of another wavelength, so light must travel into the animal and back out again, getting scattered and absorbed in both directions. Bioluminescent techniques, however, rely on chemical reactions that produce light from within the animal, so light needs to travel in only one direction. However, almost all techniques rely on transgenic cells that express the enzyme luciferase, and the light produced by bioluminescent reactions tends to be in shorter wavelengths that are more readily scattered by tissue. In a typical experiment, mice with impaired immune systems are implanted with pathogens or cancer cells that have been genetically engineered to express luciferase, then injected with the appropriate substrate, resulting in a chemical reaction that emits light.

Researchers can then create cohorts of animals or administer treatments according to how much a tumour has grown or an infection has spread. "Investigators like this because it allows you to eliminate some of the animal-to animal variation," says Stephen McAndrew of Taconic in Cranbury, New Jersey, which recently acquired the business of transgenic animals, cancer-cell lines and bacterial lines from Caliper Life Sciences in Hopkinton, Massachusetts. This can be a powerful way to show the efficacy of certain drug compounds and is regularly used in applications for the approval of investigational new drugs, he says. "You can get a lot more data points per group of animals using these technologies. My sense is that the Food and Drug Administration is very receptive."

Taconic offers nearly four dozen light-producing mice, and plans to greatly expand the types of light-emitting cancer lines it offers. And bioluminescence can be used to follow not just engineered cells but also the molecular processes within them by means of proteins called split reporters. These are used to ensure that luciferase fragments come together only when certain proteins interact or when a connecting peptide is dephosphorylated; they can also be used to monitor cell signalling or kinase activity within a living animal.

Like bioluminescent techniques, fluorescent techniques can be used for molecular imaging, such as tracking enzyme activity, or marking cells expressing a particular receptor. In this case, rather than genetically engineering cells to express a luciferase, researchers attach fluorescing dyes or nanoparticles to appropriate ligands in the laboratory, and the whole complex is then injected into the animal. One disadvantage of this method (as well as with similar labels in MRI, PET and SPECT) is that the imaging agents will also show up not just in the target site (for example, a tumour containing a particular surface receptor), but also in blood and in healthy organs. Such spurious signals can make it difficult to distinguish between, for instance, a highly vascularized site and the target. Moreover, strategies to avoid this problem can introduce other issues: increasing the dose of the imaging agent can be toxic, and waiting for the agent to clear the bloodstream and aggregate in the target zone decreases the time available for experiments.

Hisataka Kobayashi at the US National Cancer Institute in Bethesda, Maryland, grew frustrated with this problem of 'always on' labels when working with MRI and PET. So he decided to work on new probes, designing 'activatable fluorophores', which emit signals only at the desired target. Activatable fluorophores can be made in many ways, but the goal is the same, says Piwnica-Worms, who has developed similar technologies. "The signal is silent while the fluorophore is circulating around the body. You build up signal at the target and only at the target. That idea has been out there for a while, but putting it into whole-animal models is what's coming along now."

One of the most common strategies for finetuning the signal is to combine a fluorophore with a transporter tag and a quencher molecule. The transporter means that the combined molecule will be taken up only by certain types of cells. The quencher prevents fluorescence until the fluorophore binds to a cell or interacts with intercellular proteases, allowing fluorescence to occur. Kobayashi recently published work on the fluorophore indocyanine green (or ICG, which,

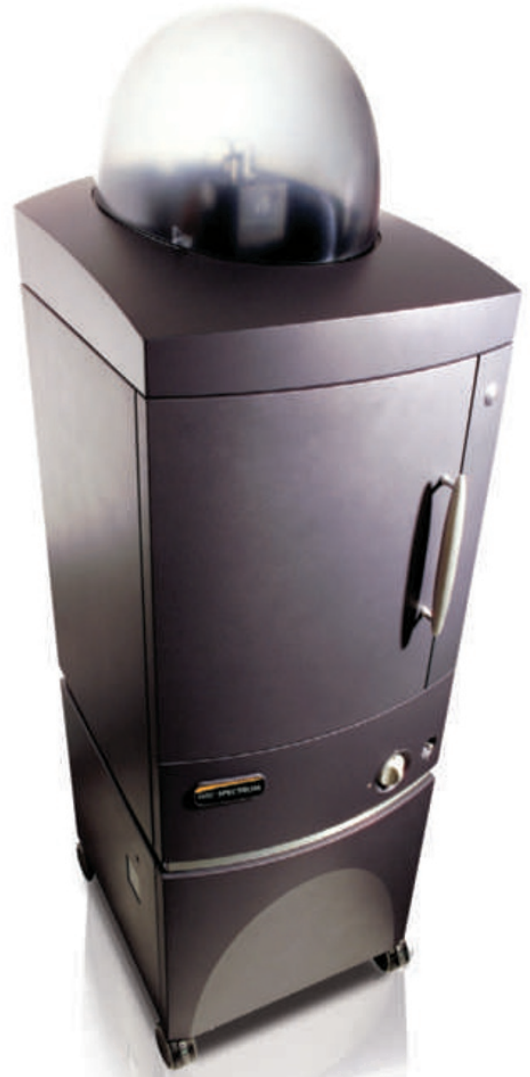

Caliper Life Sciences' IVIS Spectrum performs fluorescent and bioluminescent imaging in two and three dimensions. 
despite its name, gives off infrared light when it fluoresces). Although ICG has been clinically approved for years, it stops fluorescing when it conjugates (or covalently binds) to protein, making chemists reluctant to use it. Kobayashi reasoned that this silencing might be an advantage: he attached ICG to an antibody that both silenced the fluorescence and carried the fluorophore to the target of interest. Once the antibody was bound to the target and brought into the cell, ICG detached, becoming fluorescent ${ }^{2}$. The fact that this advantage was overlooked, says Kobayashi, is an example of how chemists' thinking can hold back development of activatable fluorophores; chemists work with such labels in solution and aren't trained to think about how fluorophores will act inside an animal's body, he says. "We pick up a lot of trash."

Probes are the workhorses of the optical imaging system, and researchers such as
Kobayashi and Piwnica-Worms are coming up with increasingly creative ways to get them into the right places and to detect them once they are there. Such advances in technology promise that researchers will soon be able to image more processes in more types of cells (see 'Probe progress'). Partly as a result, expectations for optical approaches have been rising steadily, says William McLaughlin of Carestream Molecular Imaging in Woodbridge, Connecticut, which

\section{PROBE PROGRESS}

A big limitation of molecular imaging in whole animals is how hard it is to measure multiple signals, says Sanjiv Sam Gambhir, who directs the Molecular Imaging Program at Stanford in California.

"Unlike our colleagues who remove tissues or blood samples from animals and can analyse many, many things, we're very limited in how many signals we can get simultaneously," he says. "In this field, it's not so much about the instruments but about the abilities of the imaging agents."

These agents are not always quick to develop, says Michael Olive, vice-president of science and technology at LI-COR Biosciences in Lincoln, Nebraska, which sells optical instruments and agents. It's not simply a matter of attaching a fluorophore to a ligand, he says. Before trying a new probe in an animal, for example, LI-COR uses cell lines to see whether increasing the concentration of the unlabelled ligand displaces the labelled one; if not, the label is not binding specifically and can't be used for further studies. If so, the company does more tests, then an extensive autopsy to look for any traces of the label. Without this precaution, says Olive, unanticipated labelling could wreck an experiment. "It probably takes as much as 4-6 months to have confidence that the marker is really doing what you think it's doing."

A problem for the optical techniques used to detect these probes is that most signals cannot travel very far through tissue. Some optical techniques are being used clinically, but they are restricted to organs such as the breast, bladder or stomach, where a detection device can be placed in or close to the site. By contrast, labels developed for PET or MRI for whole-body studies in patients can

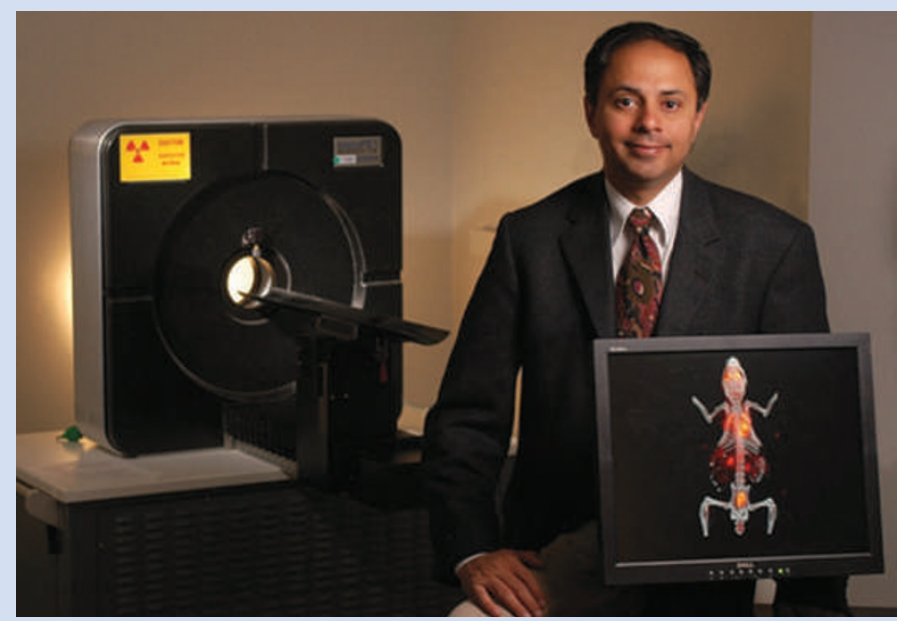

Sanjiv Sam Gambhir is working to link together various imaging modalities.

be readily used in animals. "Most of the optical technologies are never going to be translated to humans," says Michael Welch at Washington University in Saint Louis, who uses PET to study glucose metabolism in mouse models of diabetes.

Gambhir is doing his part to develop new labels. He began his career in imaging at the University of California, Los Angeles, as a teenage assistant to Michael Phelps, the co-inventor of PET. This year, he mutated luciferase to emit signals at a longer wavelength, allowing it to penetrate farther through tissues. He has also created imaging agents that produce light when acted on by particular cues, photoacoustic agents that convert light signals to sound, as well as nanoparticles that enhance Raman peaks - characteristic shifts in wavelength frequency - and so may allow detection of as many as ten spectral signals within an animal. Current versions of the particles are too large to enter cells, but Gambhir says that they can be made smaller. Nevertheless, he says, large size can be an advantage. "Delivery is harder because they are bigger, but you also get more signal because they are bigger." Several groups are working on imaging agents that are activated by one kind of energy but emit another. Fluorescent probes, for example, must be activated by light to emit light. That creates a background signal that limits the resolution of the resulting image. Optimized dyes and software can minimize the problem, but start-up nanobiotechnology firm Zymera in San Jose, California, is working on quantum dots that can bypass it. These dots are activated by high-energy, short-wavelength bioluminescent light produced within the body by luciferase coating the nanoparticle, but, rather than emitting a similar wavelength, they emit the near-infrared light best able to penetrate tissues. The dots could be used in machines optimized for fluorescence.

But getting a high-resolution image from light coming from deep within an animal, even a small one, is still a problem, says Vasilis Ntziachristos, who directs the molecular imaging department at the Technical University of Munich in Germany. Not only are many photons absorbed before they reach the charge-coupled devices that detect them, the way that tissues scatter light makes lost data extremely difficult to reconstruct. Ntziachristos is developing photoacoustic agents that create sound signals when they absorb light, and so allow signals to travel farther through tissues with less scattering. He has shown that organic dyes and fluorescent proteins can be detected with ultrasound and used for wholebody three-dimensional imaging of animals such as fruitflies, zebrafish and mice. The resolution is around 10-100 micrometres, potentially orders of magnitude better than that achievable by light, he says.

To create the signal, lasers transmit nanosecond pulses of light onto a chromophore, causing it to heat up and cool down. The resulting contraction and expansion generates a sound wave that can not only be detected but also traced to a particular depth. "When taking a photograph of an animal, what you see is the light that has been reflected or scattered through the surface," says Ntziachristos. That makes for images with poor resolution, he explains. "Photoacoustic tomography gets the resolution back."

But detecting sound rather than light is not a simple switch. Figuring out where to place the sound detectors is more complicated than setting up a home stereo system. Then there's the problem of a coupling medium, says Ntziachristos. His acoustics systems don't work as well in air, for instance. "But more importantly," he says, "you need mathematics." The data have to be processed, and variations induced by animal tissue and hardware must be accounted for. "Without the reconstruction software, you cannot produce volumetric images," he says. 
produces instruments for optical imaging. "Initially, people were seeing things they hadn't seen before, so they just wanted to see them," he recalls. Now they want to go beyond pretty pictures and actually get quantitative data, he says. Instrument-makers have to calibrate everything: from how lamps change with age, to how light criss-crosses the machine - anything that could affect the measurement.

Stephen Oldfield is the senior director of imaging marketing at Caliper, which sells the IVIS Spectrum, among other instruments. $\mathrm{He}$ thinks that three-dimensional (3D) imaging will be the next advance to become routine in optical imaging. "Early feedback was that 3D data just didn't smell right, but we can now show data that look like what you would expect from the anatomy," he says. "I think that's going to become a standard. It offers more in-depth quantification of biological events, and the ability to co-register or integrate data with other clinical 3D modalities."

\section{Multimodalities}

"Combining modalities is certainly where people have been looking most recently," says Croft. Companies such as Siemens in Berlin and Philips in Amsterdam now provide machines that combine CT with PET or SPECT. Researchers are also combining PET with MRI.

Bringing modalities together can be far
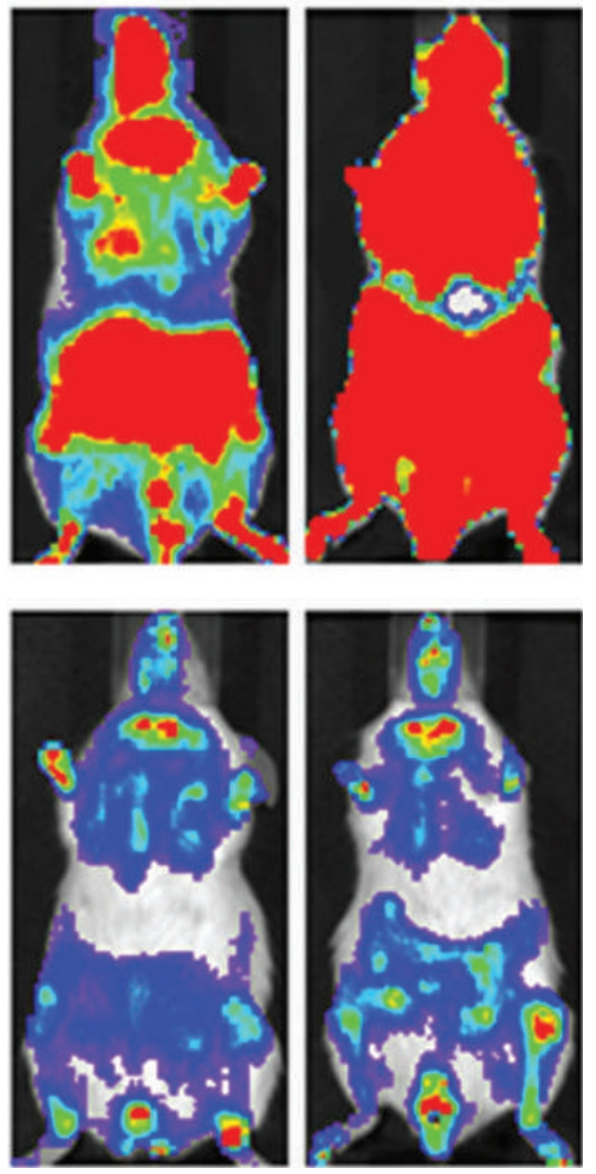

Bioluminescent $T$ cells can be used to study wayward immune reactions in disorders such as severe combined immunodeficiency disease ${ }^{4}$.

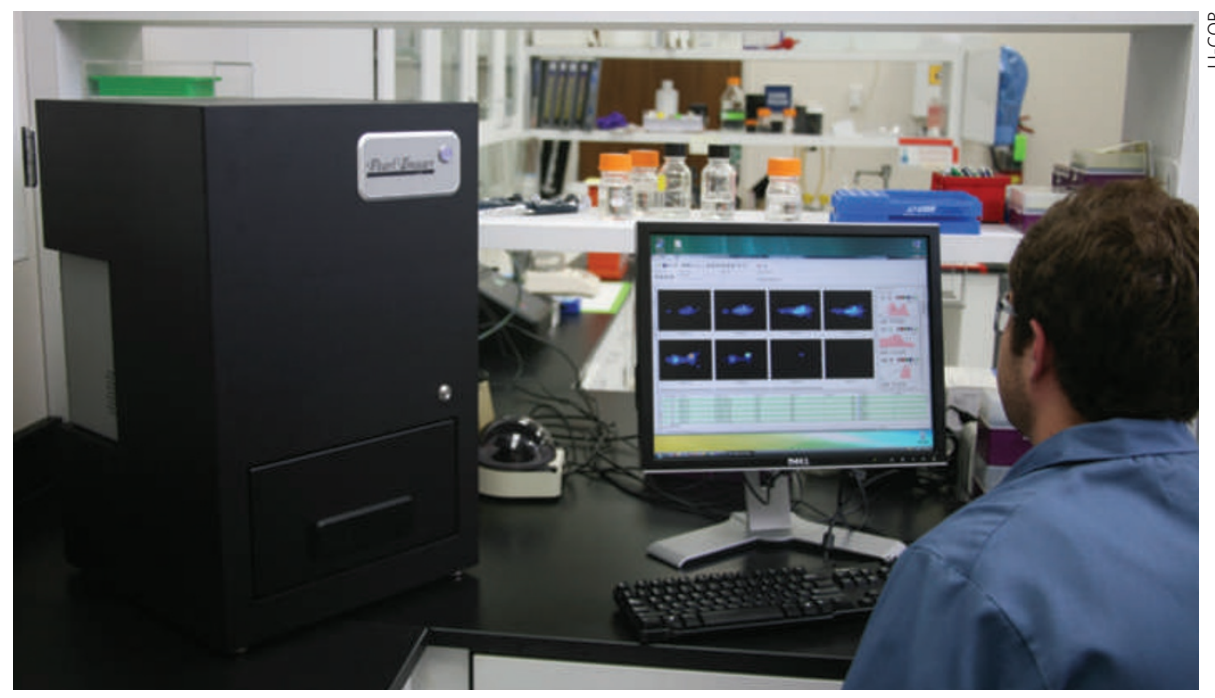

LI-COR's Pearl Imager can be used to visualize how probes target specific tissues or receptors.

from straightforward. The magnetic fields of MRI interfere with the radioactivity of PET and SPECT, for instance. Even though bioluminescence and fluorescence techniques both work by detecting light emitted from labels within the animal, fluorescent labels must first be activated by light and that must be accounted for. Moreover, the cameras need to be optimized for the different wavelengths emitted by the various labels.

Samuel Achilefu, a radiologist at Washington University in Saint Louis, is creating imaging agents designed for multimodal studies. He and his group have created an imaging probe ${ }^{3}$ that binds readily to ${ }^{64} \mathrm{Cu}$ - a radionuclide used in PET - and that contains a dye that fluoresces only after part of the probe has been cleaved by the enzyme caspase- 3 . This approach allows researchers to use PET to collect quantitative information about a disease tissue, then use the activatable fluorescent signal to detect a biomolecular event, says Achilefu, who is working to create monomolecular imaging agents that combine optical probes with probes for MRI, $\mathrm{CT}$ and ultrasound.

An alternative way to combine modalities is to create a tray, or gantry, that enables an animal to be moved between instruments without repositioning it. Systems that perform both radioisotope and X-ray imaging, for example, have trays that slide sedated animals from one detector to another. Other approaches include moulds or tubes that keep the animal still and can be moved between machines depending on what data are desired.

Furthermore, time is a factor: X-rays and optical imaging can be completed in about a minute, so both can be done in one session relatively easily. An MRI scan, however, can take hours. Combining the modalities can therefore lower throughput and extend the time that animals need to be kept under anaesthesia or are subjected to radiation, potentially harming the animal or altering the disease process.

Despite these issues, combining modalities can bring levels of clarity that had not been anticipated, says McLaughlin. Just a few years ago, researchers had to overlay optical images over an outline of the animal. Falsecolour splotches in a mouse-shaped shadow give only a general idea of where the signals are coming from, but overlaying this information with X-rays leads to a richer interpretation of the images. "It just makes it so much easier when you've got that anatomical background to be able to see where the signals are actually coming from," he says. McLaughlin recalls the reaction of researchers getting their first look at optical scans combined with X-rays about two years ago: "People would point at the images and say 'yes, yes, I understand'."

\section{Future images}

New probes and technologies allow for more sophisticated, high-definition imaging in real time. But another trend needs to be taken into account: the influx of biologists into the field. "They don't know much about imaging, but they need images," says Michael Olive, vice-president of science and technology at LI-COR Biosciences in Lincoln, Nebraska, which sells optical instruments. This is a trend that Carestream has noticed, too: in the past, the company supplied machines to laboratories with radiologists or to other imaging experts. But recently, says McLaughlin, it has started to focus on "crossing the chasm" - reaching basic-research labs in which the experience with imaging has been in microscopy rather than whole-body techniques. "We're looking at pulling some of the choices out of our system," he says. The goal is to allow researchers to study biology without needing to know what a steradian is. "It's really moving into the masses," McLaughlin says.

Monya Baker is technology editor of Nature and Nature Methods.

\footnotetext{
1. Tamgüney, G. et al. Proc. Natl Acad. Sci. USA 106, 15002-15006 (2009).

2. Ogawa, M., Kosaka, N., Choyke, P. L. \& Kobayashi, H. Cancer Res. 69, 1268-1272 (2009).

3. Lee, H. et al. J. Biomed. Opt. 14, 040507 (2009)

4. Santos, E. B. et al. Nature Med. 15, 338-344 (2009).
} 